\title{
Nobiletin Inhibits Hepatic Lipogenesis via Activation of AMP-Activated Protein Kinase
}

\author{
Taewon Yuk, ${ }^{1}$ Younghwa Kim, ${ }^{2}$ Jinwoo Yang, ${ }^{1}$ Jeehye Sung, ${ }^{1}$ \\ Heon Sang Jeong, ${ }^{1}$ and Junsoo Lee $\mathbb{D}^{1}$ \\ ${ }^{1}$ Division of Food and Animal Sciences, Chungbuk National University, Cheongju, Chungbuk 28644, Republic of Korea \\ ${ }^{2}$ School of Food Biotechnology and Nutrition, Kyungsung University, Busan 48434, Republic of Korea \\ Correspondence should be addressed to Junsoo Lee; junsoo@chungbuk.ac.kr
}

Received 29 September 2017; Revised 22 December 2017; Accepted 11 January 2018; Published 7 February 2018

Academic Editor: Luciana Dini

Copyright (C) 2018 Taewon Yuk et al. This is an open access article distributed under the Creative Commons Attribution License, which permits unrestricted use, distribution, and reproduction in any medium, provided the original work is properly cited.

\begin{abstract}
We aimed to investigate the effects of nobiletin on hepatic lipogenesis in high glucose-induced lipid accumulation in HepG2 cells. Nobiletin, a citrus polymethoxyflavonoid with six methoxy groups, is present abundantly in the peels of citrus fruits. HepG2 cells were incubated in Dulbecco's modified Eagle's medium containing high glucose $(25 \mathrm{mM})$ and subsequently treated with nobiletin at different concentrations $(5,25$, and $50 \mu \mathrm{M})$. Results showed that nobiletin markedly inhibited high glucose-induced hepatic lipid accumulation in HepG2 cells. In addition, it reduced the protein expression of lipogenic factors, including sterol regulatory elementbinding protein 1c (SREBP-1c) and fatty acid synthase (FAS). Nobiletin significantly increased the phosphorylation of AMPactivated protein kinase (AMPK) and acetyl-CoA carboxylase. Pretreatment with compound C, an AMPK inhibitor, abolished the inhibitory effects of nobiletin on SREBP-1c and FAS expression. These results suggested that nobiletin might attenuate high glucose-induced lipid accumulation in HepG2 hepatocytes via modulation of AMPK signaling pathway. Therefore, nobiletin might be useful for the prevention and treatment of nonalcoholic fatty liver diseases.
\end{abstract}

\section{Introduction}

The liver plays a key role in lipid metabolism. It is well known that a high-carbohydrate diet can prime the hepatic de novo lipogenesis (DNL) pathway with a large substrate load and, thus, increase the rate of DNL [1]. Accumulation of lipid droplets in the hepatocytes has been closely related to obesity, insulin resistance, type 2 diabetes, and nonalcoholic fatty liver diseases (NAFLD) [2,3]. Elevated hepatic DNL was observed in insulin resistance condition [4-6]; therefore, a finding of high DNL rate in an individual may provide early warning of the possible development of type 2 diabetes mellitus. Therefore, the prevention of increased DNL in the liver may provide a therapeutic strategy for the management of multiple stages of NAFLD.

Hepatic AMP-activated protein kinase (AMPK) is thought to play a pivotal role in regulating lipid metabolism, glucose homeostasis, and insulin sensitivity $[7,8]$. As an energy sensor maintaining cellular glucose homeostasis, AMPK significantly inhibits hepatic glucose output by transcriptional control [9]. In addition, AMPK regulates acyl coenzyme A (acyl-CoA) channeling towards $\beta$-oxidation and away from glycerolipid biosynthesis. AMPK phosphorylates and downregulates acetyl-CoA carboxylase (ACC), thereby decreasing the production of malonyl-CoA. Sterol regulatory elementbinding proteins (SREBPs) are key lipogenic transcription factors that regulate the expression of lipogenic enzymes, including ACC, fatty acid synthase (FAS), and 3-hydroxy3 -methylglutaryl-CoA reductase [10-12]. Overexpression of hepatic SREBP-1c results in selective induction of lipogenic gene expression, with no effect on the genes involved in cholesterol synthesis [13]. Moreover, the phosphorylation of AMPK inactivates SREBP-1 and inhibits hepatic lipid accumulation in a high-fat diet-induced mouse [14, 15].

Nobiletin, a major component in citrus fruits [16], has been used in traditional Chinese herbal medicine [17]. It has been shown to possess several biological activities, including anti-inflammatory [18], antitumor [19], antidiabetic [20], and antiobesity [21] effects, as well as play an important role in the prevention of postprandial hyperglycemia [22] and 
<smiles>COc1ccc(-c2cc(=O)c3c(OC)c(OC)c(OC)c(OC)c3o2)cc1OC</smiles>

FIGURE 1: Structure of nobiletin.

hyperlipidemia [23]. However, the molecular mechanisms underlying its effects on hepatic lipid metabolism, particularly with regard to the activation of AMPK, are still unclear. In this study, we investigated the antilipogenic effects of nobiletin on high glucose-induced lipid accumulation in HepG2 cells through the AMPK signaling pathway.

\section{Materials and Methods}

2.1. Chemicals. Nobiletin (purity $\geq 95 \%$ ) was purchased from Wako Pure Chemicals (Osaka, Japan) (Figure 1). Dulbecco's modified Eagle's medium (DMEM), fetal bovine serum (FBS), trypsin-ethylenediaminetetraacetic acid (EDTA), and penicillin-streptomycin were obtained from Gibco BRL (Grand Island, NY, USA). 6-[4-(2-Piperidin-1-ylethoxy)phenyl]-3pyridin-4-ylpyrazolo[1,5-a]pyrimidine (Compound C), 3(4,5-dimethylthiazol-2-yl)-2,5-diphenyltetrazolium (MTT), dimethyl sulfoxide (DMSO), lactate dehydrogenase (LDH) kit, and Oil red O (ORO) were purchased from SigmaAldrich (St. Louis, MO, USA). Antibodies against p-AMPK, p-ACC, AMPK, and ACC were obtained from Cell Signaling Technology (Beverly, MA, USA). Antibodies against SREBP1c, FAS, and $\beta$-actin were purchased from Santa Cruz Biotechnology (Santa Cruz, CA, USA). Enhanced chemiluminescence $\left(\mathrm{ECL}^{\mathrm{TM}}\right)$ detection reagents were purchased from GE Healthcare (Buckinghamshire, UK). All other reagents and solvents were of analytical and HPLC grades.

2.2. HepG2 Cell Culture and Hepatic Lipogenesis. HepG2 cells were obtained from the Korean Collection for Type Cultures (KCTC, Daejeon, Korea) and cultured in DMEM supplemented with $10 \%$ heat-inactivated FBS, $100 \mathrm{U} / \mathrm{mL}$ penicillin, $100 \mu \mathrm{g} / \mathrm{mL}$ streptomycin, and $5.5 \mathrm{mM}$ D-glucose. HepG2 cells were maintained in a humidified incubator with $5 \% \mathrm{CO}_{2}$ at $37^{\circ} \mathrm{C}$.

A cell model of high glucose-induced lipid accumulation in hepatocytes was prepared by exposing HepG2 cells to a high concentration of glucose $(25 \mathrm{mM})$ for $24 \mathrm{~h}$, as previously described [24]. Briefly, HepG2 cells were cultured in FBS-free medium overnight to induce serum depletion. Then, HepG2 cells were incubated in FBS-free medium containing high concentration of glucose $(25 \mathrm{mM})$ and subsequently treated with nobiletin at different concentrations $(5,25$, and $50 \mu \mathrm{M})$.

2.3. Cytotoxicity. After $24 \mathrm{~h}$, MTT reagent $(0.5 \mathrm{mg} / \mathrm{mL})$ dissolved in FBS-free DMEM was added to each well, and the cells were incubated for $3 \mathrm{~h}$ at $37^{\circ} \mathrm{C}$. The culture medium was removed, and the intracellular formazan product was dissolved by adding DMSO. The absorbance was measured at $550 \mathrm{~nm}$ using a spectrophotometer (BioTek Instruments, Inc., Winooski, VT, USA).

2.4. Oil Red O Staining. To measure intracellular lipid accumulation, HepG2 cells were stained by ORO. HepG2 cells were cultured in FBS-free medium overnight to induce serum depletion. After $24 \mathrm{~h}, \mathrm{HepG} 2$ cells were incubated in FBS-free medium containing either normal $(5.5 \mathrm{mM})$ or high $(25 \mathrm{mM})$ concentration of D-glucose and subsequently treated with nobiletin at different concentrations. After treatment, HepG2 cells were washed twice with cold phosphate-buffered saline (PBS) and fixed with $10 \%$ formaldehyde for $10 \mathrm{~min}$. The fixed cells were washed three times with distilled water and stained with ORO solution (stock solution, $3 \mathrm{mg} / \mathrm{mL}$ in isopropanol; working solution, $60 \%$ ORO stock solution and $40 \%$ distilled water) for $30 \mathrm{~min}$ at room temperature. The cells were rinsed three times with distilled water. Finally, the ORO dye retained in the cells was eluted with isopropanol and quantified by measuring its absorbance at $500 \mathrm{~nm}$.

2.5. Western Blot Analysis. To measure the expression of lipid accumulation-related proteins, western blot analysis was performed. HepG2 cells were washed with cold PBS and then collected by centrifugation. The washed cell pellets were lysed in a cell lysis buffer (iNtRON Biotech, Seongnam, Korea), according to the manufacturer's instructions. The protein concentration was determined using Take $3^{\mathrm{TM}}$ Multi-Volume Plate model in an Epoch plate reader (BioTek Instruments, Inc.) and calculated by Gen5 software (BioTek Instruments, Inc.). Proteins were separated by $10 \%$ sodium dodecyl sulfate(SDS-) polyacrylamide gel and transferred onto nitrocellulose membranes. The membranes were blocked in a blocking solution (5\% skim milk in Tris-buffered saline/Tween 20 [TBST]) for $1 \mathrm{~h}$ at room temperature. They were incubated for $3 \mathrm{~h}$ with polyclonal antibodies against $\mathrm{p}$-AMPK, $\mathrm{p}$-ACC, AMPK, ACC, FAS, SREBP- $1 c$, and monoclonal anti- $\beta$-actin antibodies (1:1000 each) in TBST containing 5\% skim milk. The membranes were then washed three times with TBST and incubated with horseradish-conjugated secondary antibody $(1: 1000)$ for $1 \mathrm{~h}$ at room temperature. The membranes were again washed three times with TBST. The specific protein bands were visualized on an X-ray film activated by chemiluminescence using ECL detection reagents. The autoradiograms were quantified by optical densitometry using ImageJ software (NIH, Washington, DC, USA).

2.6. Statistical Analysis. Data were expressed as the means \pm standard errors (SE) of at least three independent experiments. Statistical analysis was performed by Duncan's test using SAS version 9.4 (SAS Institute, Inc., Cary, NC, USA). A $p$ value $<0.05$ was considered statistically significant.

\section{Results and Discussion}

3.1. Effects of Nobiletin on Cytotoxicity and Lipid Accumulation. MTT assay was used to determine the effects of nobiletin on the viability of HepG2 cells. Cells were incubated in the presence or absence of nobiletin in DMEM containing 


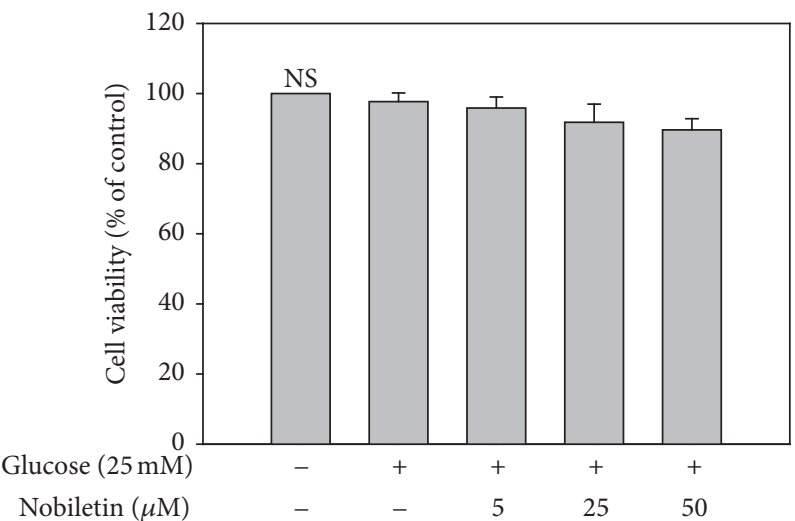

(a)
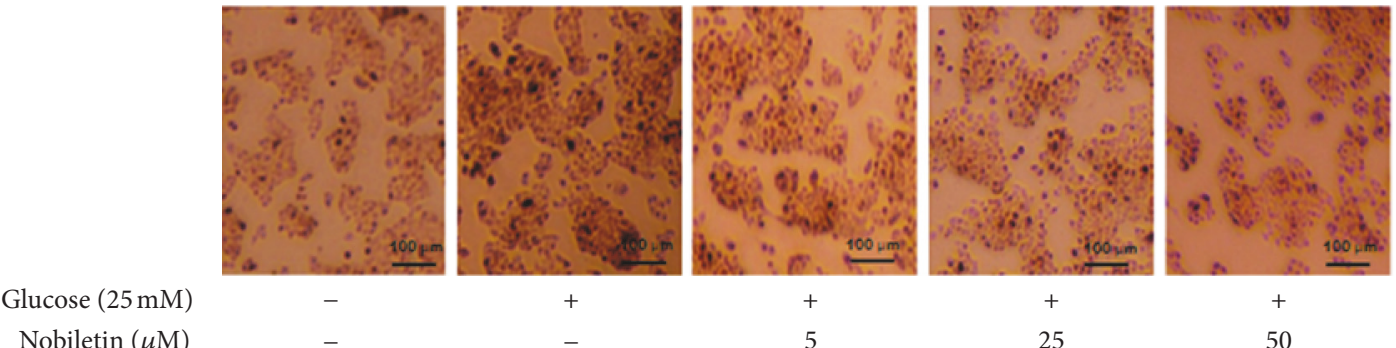

Nobiletin $(\mu \mathrm{M})$

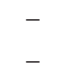

$+$

$+$

25

50

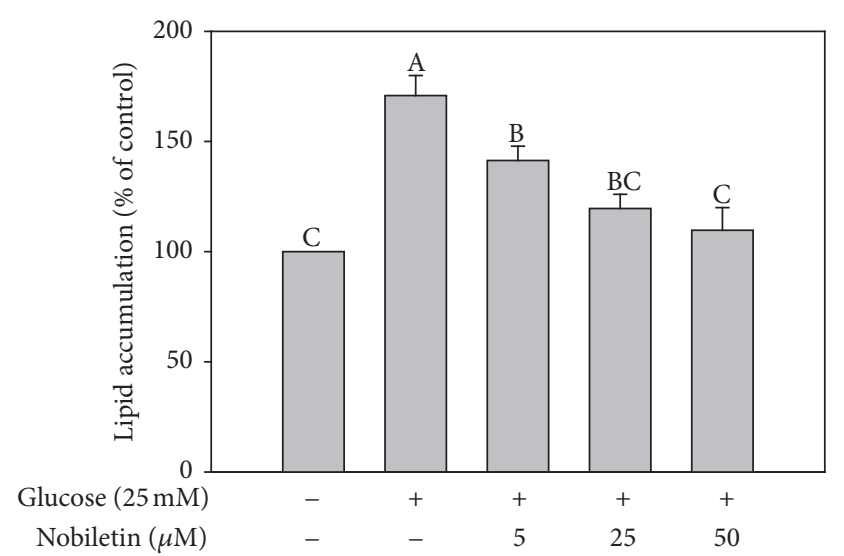

(b)

FIGURE 2: Effects of nobiletin on cytotoxicity (a) and lipid accumulation (b) in HepG2 cells. HepG2 cells were treated with high glucose $(25 \mathrm{mM})$ and different concentrations of nobiletin for $24 \mathrm{~h}$. Values are expressed as the means \pm standard errors $(n=3)$. Different letters indicate a significant difference at $p<0.05$. NS: nonsignificant difference between the control and all treatments $(p<0.05)$.

high concentration of glucose $(25 \mathrm{mM})$ for $24 \mathrm{~h}$. As shown in Figure 2(a), no cytotoxicity was observed at any of the concentrations examined $(5,25$, and $50 \mu \mathrm{M})$. These results suggested that nobiletin did not affect the viability of HepG2 cells.

To examine the effects of nobiletin on lipid accumulation, HepG2 cells were cultured in DMEM containing high concentration of glucose $(25 \mathrm{mM})$ and various concentrations of nobiletin $(5,25$, and $50 \mu \mathrm{M})$ for $24 \mathrm{~h}$. ORO staining was performed to measure the total lipid accumulation in HepG2 cells. Nobiletin significantly reduced high glucoseinduced intracellular lipid accumulation (Figure 2(b)). In line with these findings, a previous study showed that nobiletin suppressed lipid accumulation in 3T3-L1 cells [21]. In addition, nobiletin attenuated the activity of glycerol 3-phosphate dehydrogenase (GPDH) [21], which plays an important role in the conversion of glycerol to triglycerides [25]. The results of the present study showed that nobiletin exhibited inhibitory effects against lipid accumulation in HepG2 cells.

3.2. Effects of Nobiletin on SREBP-1c and FAS Expression. Nobiletin treatment decreased the protein expression of SREBP-1c (Figure 3(a)) and FAS (Figure 3(b)) in high glucosestimulated HepG2 cells in a concentration-dependent manner. The regulation of lipogenic gene expression by insulin and fatty acids is mainly mediated by transcription factors, such as SREBPs [26]. SREBP-1c, a glucose-dependent transcription factor, plays a crucial role in the regulation of the expression of lipogenic genes, such as FAS, in the liver [27]. Overexpression of genes that activate lipogenesis, such as SREBP-1, FAS, and ACC, has been observed in steatotic livers 

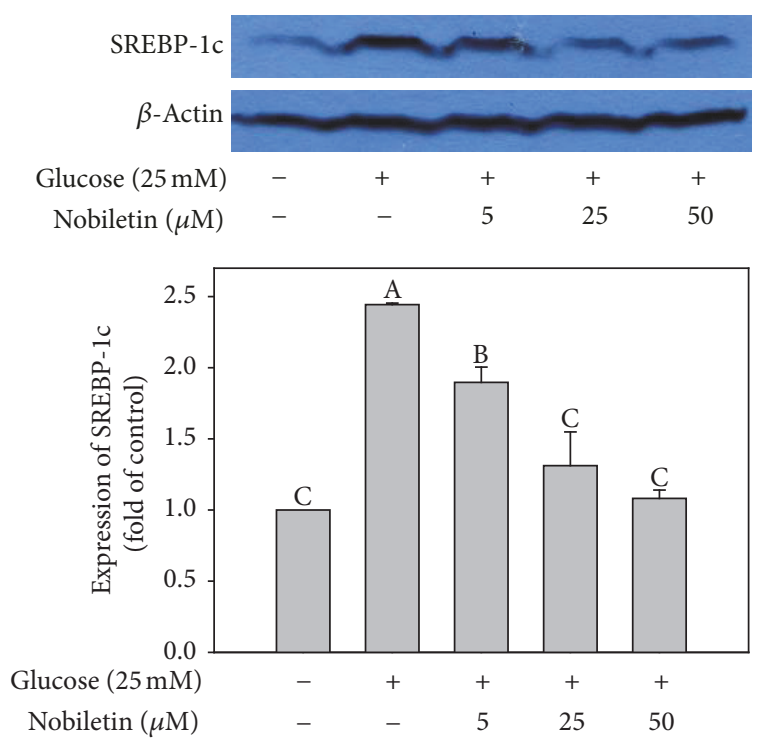

(a)
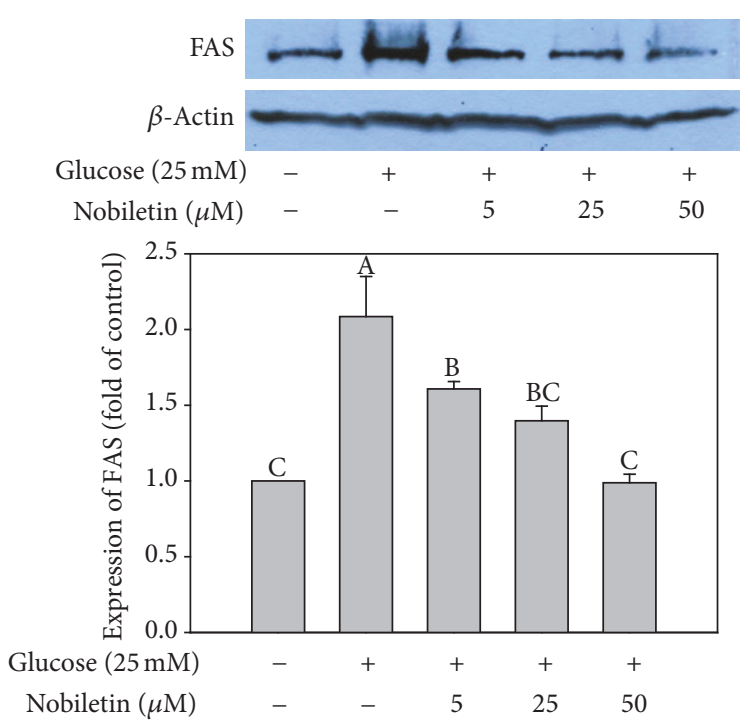

(b)

FIGURE 3: Effects of nobiletin on SREBP-1c (a) and FAS (b) expression in HepG2 cells. HepG2 cells were treated with high glucose (25 mM) and different concentrations of nobiletin for $24 \mathrm{~h}$. Values are expressed as the means \pm standard errors $(n=3)$. Different letters indicate a significant difference at $p<0.05$. Blots are representative of at least three independent experiments.
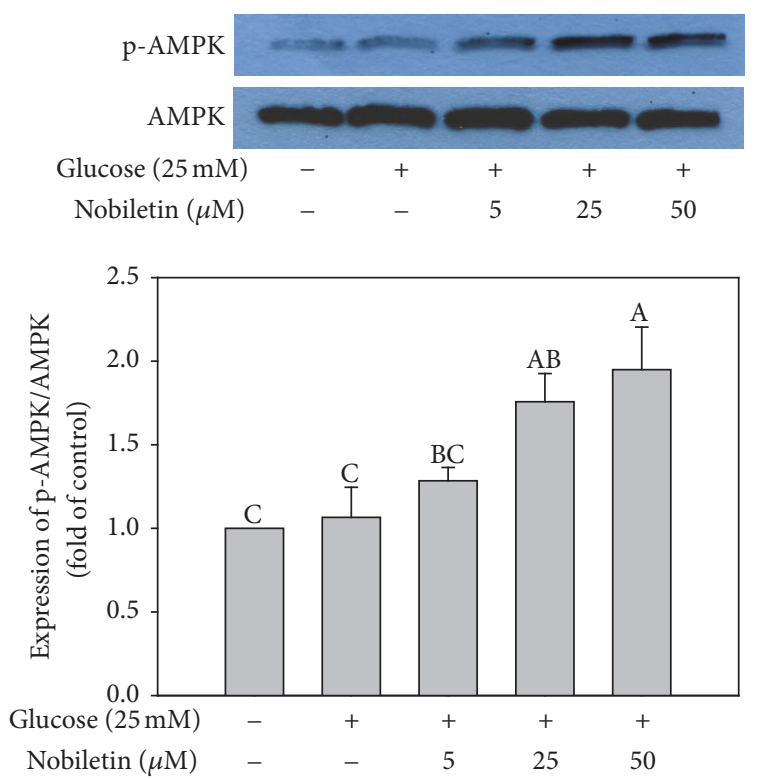

(a)
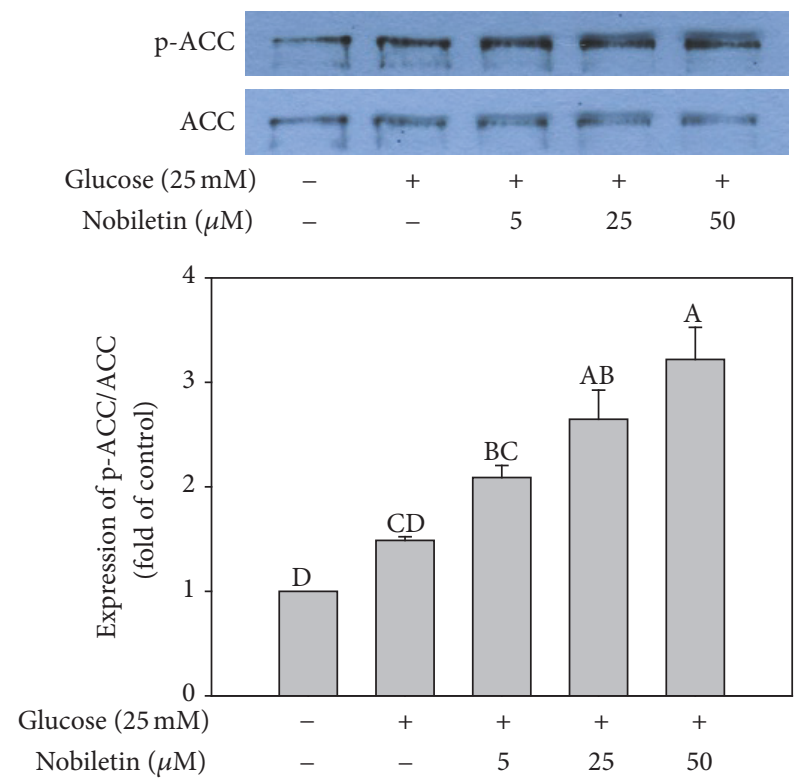

(b)

FIGURE 4: Effects of nobiletin on AMPK (a) and ACC (b) phosphorylation in HepG2 cells. HepG2 cells were treated with high glucose (25 mM) and different concentrations of nobiletin for $24 \mathrm{~h}$. Values are expressed as the means \pm standard errors $(n=3)$. Different letters indicate a significant difference at $p<0.05$. Blots are representative of at least three independent experiments.

of humans and obese mice $[28,29]$. These results indicated that nobiletin suppressed lipid accumulation via inhibition of the expression of lipogenic proteins, such as SREBP-1c and FAS, in HepG2 cells. In addition, these findings suggested that SREBP-1c and FAS might be the targets of nobiletin with regard to its hypolipidemic effects in hepatocytes.

3.3. Effects of Nobiletin on Phosphorylation of AMPK and ACC. To investigate the effect on the phosphorylation of AMPK and
ACC by high glucose conditions, HepG2 cells were incubated in serum-free DMEM containing high concentration of glucose $(25 \mathrm{mM})$ in the absence or presence of nobiletin (5, 25 , and $50 \mu \mathrm{M})$ for $24 \mathrm{~h}$. Nobiletin treatment significantly increased the expression of p-AMPK and p-ACC in a concentration-dependent manner (Figures 4(a) and 4(b)). It has been shown that AMPK modulates several key lipid metabolism-related transcription factors, including SREBP1c [30]. The activation of AMPK suppresses SREBP-1c 

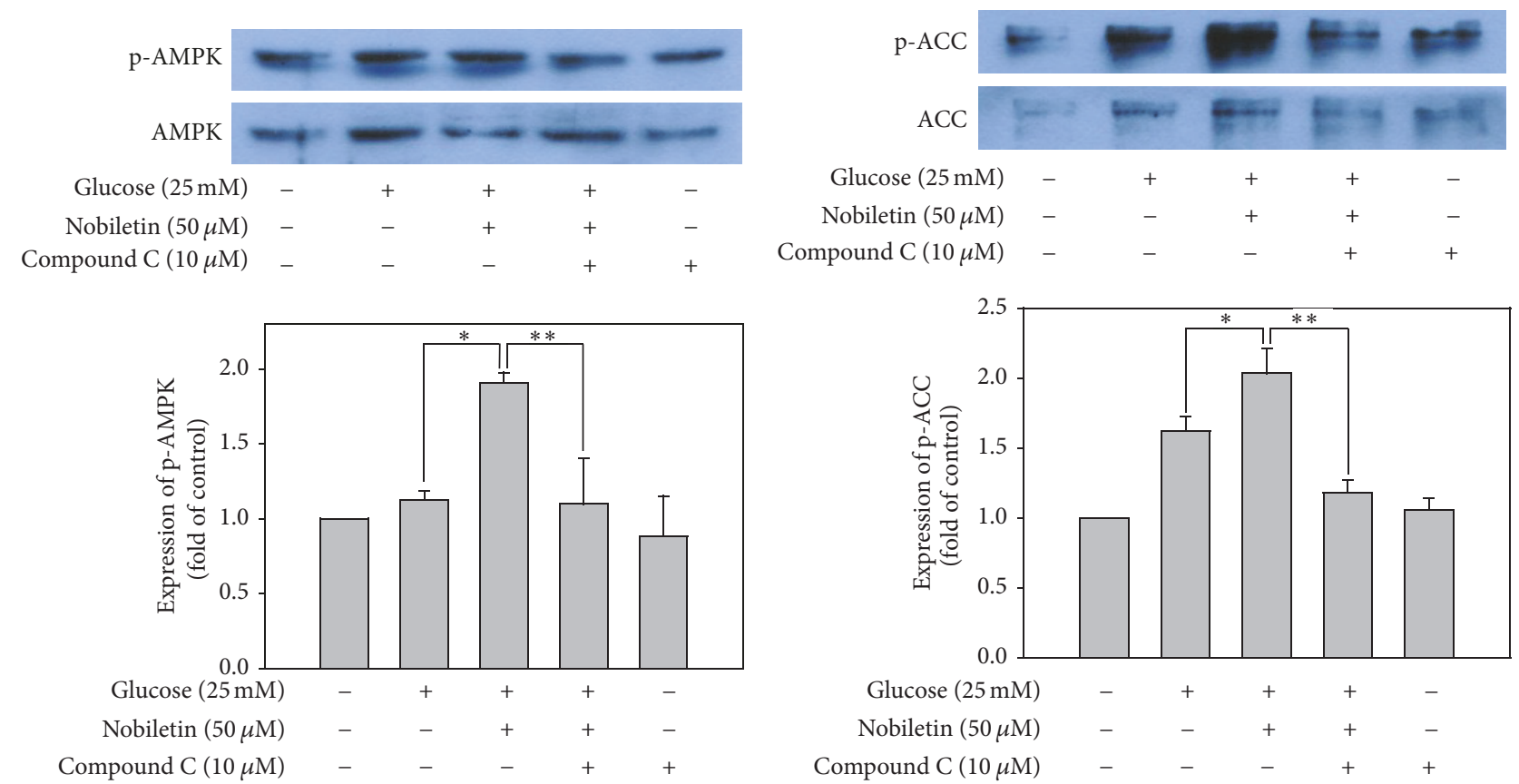

(a)
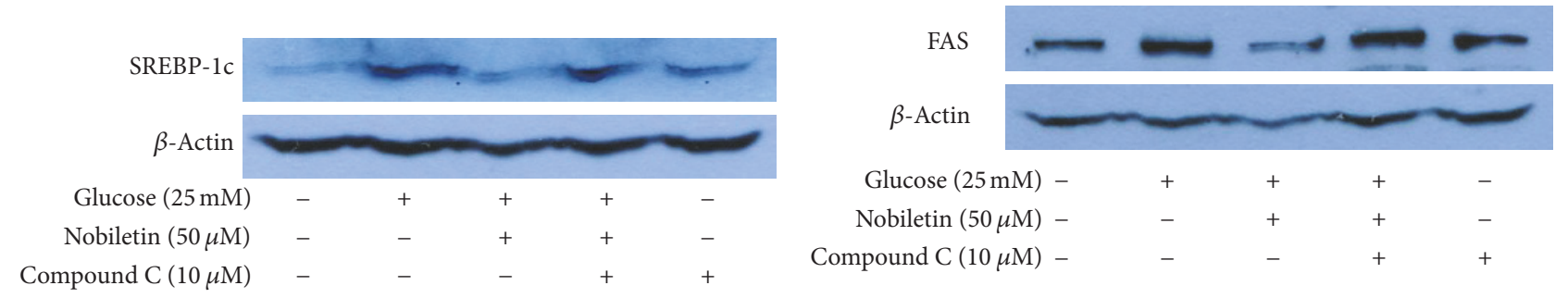

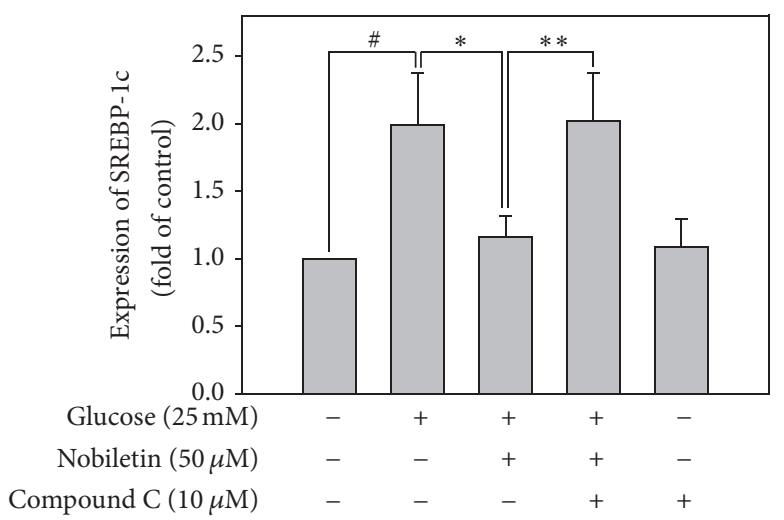

(c)

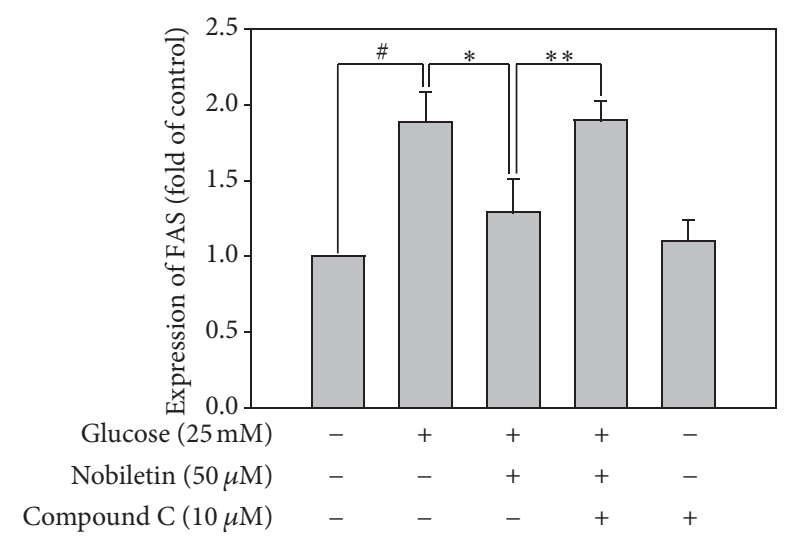

(d)

FIGURE 5: Involvement of AMPK in the inhibitory effects of nobiletin on lipid accumulation. The protein expression levels of phosphorylated AMPK (a), phosphorylated ACC (b), SREBP-1c (c), and FAS (d). Values are expressed as the means \pm standard errors $(n=3)$. * $p<0.05$ versus high glucose-treated cells; ${ }^{*} p<0.05$ versus control; ${ }^{* *} p<0.05$ versus high glucose plus $50 \mu \mathrm{M}$ nobiletin-treated cells. Blots are representative of at least three independent experiments.

expression [31] and inhibits its proteolytic processing and transcriptional activity [30]. 5-Aminoimidazole-4carboxamide-riboside (AICAR), an AMPK activator, was shown to inhibit adipocyte differentiation and block the expression of late adipogenic markers, such as peroxisome proliferator-activated receptor gamma (PPAR $\gamma)$ and CCAAT-enhancer-binding protein alpha $(\mathrm{C} / \mathrm{EBP} \alpha)$ in adipocytes [32]. In a previous study, once the suppression of AMPK signaling was compensated, the increase in lipid accumulation was obviously abolished [33]. These results 
showed that activation of AMPK by nobiletin might, in part, be involved in the inhibition of lipid accumulation in HepG2 cells.

\subsection{Involvement of AMPK in the Inhibitory Effects of Nobiletin} on Lipid Accumulation. To confirm the role of AMPK in the regulation of lipid metabolic gene expression, HepG2 cells were treated with $10 \mu \mathrm{M}$ compound $\mathrm{C}$, an AMPK inhibitor, in serum-free medium for $1 \mathrm{~h}$, and then incubated with or without $50 \mu \mathrm{M}$ nobiletin in DMEM containing high concentration of glucose $(25 \mathrm{mM})$ for an additional $24 \mathrm{~h}$. The increase in p-AMPK and p-ACC expression by nobiletin was completely abolished by compound C pretreatment in HepG2 cells (Figures 5(a) and 5(b)). These results indicated that nobiletin induced ACC phosphorylation by activating AMPK signaling pathway in HepG2 cells.

To elucidate whether inhibition of several key lipid metabolism-related transcription factors by nobiletin might be regulated by AMPK activation, the protein expression of SREBP-1c and its target lipogenic enzymes, such as FAS, was evaluated. Although nobiletin significantly reduced the expression of SREBP-1c and FAS in high glucose-treated HepG2 cells, compound C pretreatment abolished these inhibitory effects of nobiletin (Figures 5(c) and 5(d)). AMPK activation decreases lipogenesis by inhibiting SREBP-1c activation, thereby downregulating ACC and FAS expression [14]. AMPK directly phosphorylates SREBP-1c and SREBP-2. It was shown to stimulate Ser372 phosphorylation, suppress SREBP-1c cleavage and nuclear translocation, and repress SREBP-1c target gene expression in hepatocytes exposed to high glucose concentrations, resulting in reduced lipogenesis and lipid accumulation [30]. These results suggested that nobiletin could inhibit de novo lipogenesis via downregulation of lipogenic gene transcription in HepG2 cells by activating the AMPK signaling pathway.

In addition, the effects of AMPK on the regulation of intracellular lipid accumulation were investigated in HepG2 cells. Total lipid content was measured by ORO staining. Lipid accumulation significantly decreased in high glucosestimulated HepG2 cells treated with nobiletin; however, compound $\mathrm{C}$ increased lipid accumulation in HepG2 cells (Figure 6). A previous study showed that nobiletin markedly inhibited adipogenesis of preadipocytes in mature adipocytes by blocking the expression of the adipogenic transcription factors, such as PPAR $\gamma$ and $\mathrm{C} / \mathrm{EBP} \alpha$ via activation of AMPK signaling pathway [21]. Moreover, various natural compounds, such as gomisin $\mathrm{N}$ and genistein, were shown to exert protective effects against hepatic steatosis by inhibiting lipogenesis and stimulating fatty acid oxidation through AMPK activation in vivo $[34,35]$. Taken together, these results showed that nobiletin reduced total lipid content by activating AMPK signaling pathway in HepG2 cells.

\section{Conclusions}

In conclusion, we investigated the molecular mechanisms, by which nobiletin attenuated intracellular lipid accumulation in HepG2 cells. Nobiletin decreased SREBP-1c and FAS

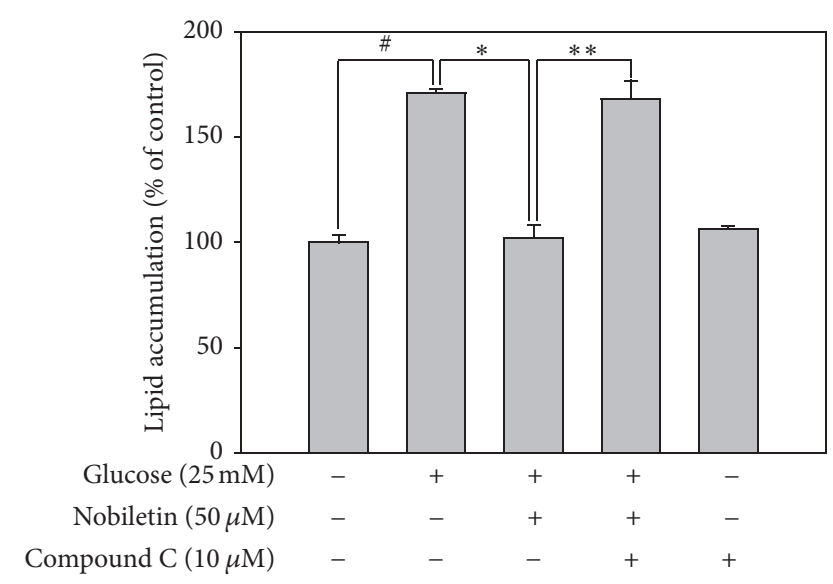

FIGURE 6: Involvement of AMPK pathway in the inhibitory effects of nobiletin and compound C, an AMPK inhibitor, on lipid accumulation in HepG2 cells. Quantification of accumulated lipids was measured by Oil red $\mathrm{O}$ staining. Values are expressed as the means \pm standard errors $(n=3) .{ }^{\#} p<0.05$ versus the control cells, ${ }^{*} p<0.05$ versus high glucose-treated cells, and ${ }^{* *} p<0.05$ versus high glucose plus $50 \mu \mathrm{M}$ nobiletin-treated cells.

expression via activation of AMPK signaling pathway. To the best of our knowledge, this is the first study showing that AMPK activation by nobiletin attenuated lipid accumulation in HepG2 cells. Collectively, these results suggested that nobiletin might have been a potential functional food ingredient for preventing liver diseases, such as hyperlipidemia and hepatic steatosis.

\section{Disclosure}

An earlier version of this work was presented as a poster at the Experimental Biology 2015 Annual Meeting (The FASEB Journal vol. 29, no. 1 Supplement 917.4).

\section{Conflicts of Interest}

The authors declare that there are no conflicts of interest regarding the publication of this paper.

\section{Acknowledgments}

This research was supported by the Basic Science Research Program through the National Research Foundation of Korea (NRF) funded by the Ministry of Education, Science, and Technology (Project no. 2017R1D1A1B03036360), Republic of Korea.

\section{References}

[1] J.-M. Schwarz, P. Linfoot, D. Dare, and K. Aghajanian, "Hepatic de novo lipogenesis in normoinsulinemic and hyperinsulinemic subjects consuming high-fat, low-carbohydrate and lowfat, high-carbohydrate isoenergetic diets," American Journal of Clinical Nutrition, vol. 77, no. 1, pp. 43-50, 2003.

[2] G. Marchesini, M. Brizi, G. Blanchi et al., "Nonalcoholic fatty liver disease: a feature of the metabolic syndrome," Diabetes, vol. 50, no. 8, pp. 1844-1850, 2001. 
[3] P. Nguyen, V. Leray, M. Diez et al., "Liver lipid metabolism," Journal of Animal Physiology and Animal Nutrition, vol. 92, no. 3, pp. 272-283, 2008.

[4] L. A. Adams, A. Feldstein, K. D. Lindor, and P. Angulo, "Nonalcoholic fatty liver disease among patients with hypothalamic and pituitary dysfunction," Hepatology, vol. 39, no. 4, pp. 909914, 2004.

[5] D. Azzout-Marniche, D. Becard, C. Guichard, M. Foretz, P. Ferre, and F. Foufelle, "Insulin effects on sterol regulatoryelement-binding protein-1c (SREBP-1c) transcriptional activity in rat hepatocytes," Biochemical Journal, vol. 350, no. 2, pp. 389393, 2000.

[6] G. F. Lewis, A. Carpentier, K. Adeli, and A. Giacca, "Disordered fat storage and mobilization in the pathogenesis of insulin resistance and type 2 diabetes," Endocrine Reviews, vol. 23, no. 2, pp. 201-229, 2002.

[7] D. Carling, "AMP-activated protein kinase: Balancing the scales," Biochimie, vol. 87, no. 1, pp. 87-91, 2005.

[8] B. B. Kahn, T. Alquier, D. Carling, and D. G. Hardie, "AMPactivated protein kinase: ancient energy gauge provides clues to modern understanding of metabolism," Cell Metabolism, vol. 1, no. 1, pp. 15-25, 2005.

[9] H.-Y. Quan, H.-D. Yuan, M. I. S. Jung, S. K. Ko, Y. G. Park, and S. H. Chung, "Ginsenoside Re lowers blood glucose and lipid levels via activation of AMP-activated protein kinase in HepG2 cells and high-fat diet fed mice," International Journal of Molecular Medicine, vol. 29, no. 1, pp. 73-80, 2011.

[10] J. L. Goldstein, R. B. Rawson, and M. S. Brown, "Mutant mammalian cells as tools to delineate the sterol regulatory element-binding protein pathway for feedback regulation of lipid synthesis," Archives of Biochemistry and Biophysics, vol. 397, no. 2, pp. 139-148, 2002.

[11] J. D. Horton, "Sterol regulatory element-binding proteins: transcriptional activators of lipid synthesis," Biochemical Society Transactions, vol. 30, no. 6, pp. 1091-1095, 2002.

[12] J. L. Goldstein and M. S. Brown, "From fatty streak to fatty liver: 33 Years of joint publications in the JCI," The Journal of Clinical Investigation, vol. 118, no. 4, pp. 1220-1222, 2008.

[13] D. Eberlé, B. Hegarty, P. Bossard, P. Ferré, and F. Foufelle, "SREBP transcription factors: Master regulators of lipid homeostasis," Biochimie, vol. 86, no. 11, pp. 839-848, 2004.

[14] Y. W. Kim, Y. M. Kim, Y. M. Yang et al., "Inhibition of SREBP-1cmediated hepatic steatosis and oxidative stress by sauchinone, an AMPK-activating lignan in Saururus chinensis," Free Radical Biology \& Medicine, vol. 48, no. 4, pp. 567-578, 2010.

[15] S.-L. Hwang, Y.-T. Jeong, X. Li et al., "Inhibitory cross-talk between the AMPK and ERK pathways mediates endoplasmic reticulum stress-induced insulin resistance in skeletal muscle," British Journal of Pharmacology, vol. 169, no. 1, pp. 69-81, 2013.

[16] Y. Nogata, K. Sakamoto, H. Shiratsuchi, T. Ishii, M. Yano, and H. Ohta, "Flavonoid composition of fruit tissues of citrus species," Bioscience, Biotechnology, and Biochemistry, vol. 70, no. 1, pp. 178-192, 2006.

[17] J. A. Manthey, T. B. Cesar, E. Jackson, and S. MertensTalcott, "Pharmacokinetic study of nobiletin and tangeretin in rat serum by high-performance liquid chromatographyelectrospray ionization-mass spectrometry," Journal of Agricultural and Food Chemistry, vol. 59, no. 1, pp. 145-151, 2011.

[18] Y.-Q. Wu, C.-H. Zhou, J. Tao, and S.-N. Li, "Antagonistic effects of nobiletin, a polymethoxyflavonoid, on eosinophilic airway inflammation of asthmatic rats and relevant mechanisms," Life Sciences, vol. 78, no. 23, pp. 2689-2696, 2006.
[19] Y. Miyata, T. Sato, M. Yano, and A. Ito, "Activation of protein kinase $\mathrm{C} \beta \mathrm{II} / \mathrm{E}-\mathrm{c}-J u n \mathrm{NH} 2$-terminal kinase pathway and inhibition of mitogen-activated protein/extracellular signalregulated kinase 1/2 phosphorylation in antitumor invasive activity induced by the polymethoxy flavonoid, nobiletin," Molecular Cancer Therapeutics, vol. 3, no. 7, pp. 839-847, 2004.

[20] Y.-S. Lee, B.-Y. Cha, K. Saito et al., "Nobiletin improves hyperglycemia and insulin resistance in obese diabetic ob/ob mice," Biochemical Pharmacology, vol. 79, no. 11, pp. 1674-1683, 2010.

[21] Y. Choi, Y. Kim, H. Ham, Y. Park, H.-S. Jeong, and J. Lee, "Nobiletin suppresses adipogenesis by regulating the expression of adipogenic transcription factors and the activation of AMPactivated protein kinase (AMPK)," Journal of Agricultural and Food Chemistry, vol. 59, no. 24, pp. 12843-12849, 2011.

[22] W. Shen, Y. Xu, and Y.-H. Lu, "Inhibitory effects of Citrus flavonoids on starch digestion and antihyperglycemic effects in HepG2 cells," Journal of Agricultural and Food Chemistry, vol. 60, no. 38, pp. 9609-9619, 2012.

[23] S. Miyamoto, Y. Yasui, T. Tanaka, H. Ohigashi, and A. Murakami, "Suppressive effects of nobiletin on hyperleptinemia and colitis-related colon carcinogenesis in male ICR mice," Carcinogenesis, vol. 29, no. 5, pp. 1057-1063, 2008.

[24] M. Zang, A. Zuccollo, X. Hou et al., "AMP-activated protein kinase is required for the lipid-lowering effect of metformin in insulin-resistant human HepG2 cells," The Journal of Biological Chemistry, vol. 279, no. 46, pp. 47898-47905, 2004.

[25] L. S. Wise and H. Green, "Participation of one isozyme of cytosolic glycerophosphate dehydrogenase in the adipose conversion of 3T3 cells," The Journal of Biological Chemistry, vol. 254, no. 2, pp. 273-275, 1979.

[26] H.-J. Kim, M. Miyazaki, W. C. Man, and J. M. Ntambi, "Sterol regulatory element-binding proteins (SREBPs) as regulators of lipid metabolism: polyunsaturated fatty acids oppose cholesterol-mediated induction of SREBP-1 maturation," Annals of the New York Academy of Sciences, vol. 967, no. 1, pp. 34-42, 2002.

[27] H. Shimano, N. Yahagi, M. Amemiya-Kudo et al., "Sterol regulatory element-binding protein-1 as a key transcription factor for nutritional induction of lipogenic enzyme genes," The Journal of Biological Chemistry, vol. 274, no. 50, pp. 3583235839, 1999.

[28] B. K. Straub, P. Stoeffel, H. Heid, R. Zimbelmann, and P. Schirmacher, "Differential pattern of lipid droplet-associated proteins and de novo perilipin expression in hepatocyte steatogenesis," Hepatology, vol. 47, no. 6, pp. 1936-1946, 2008.

[29] M. Bell, H. Wang, H. Chen et al., "Consequences of lipid droplet coat protein downregulation in liver cells: abnormal lipid droplet metabolism and induction of insulin resistance," Diabetes, vol. 57, no. 8, pp. 2037-2045, 2008.

[30] Y. Li, S. Xu, M. M. Mihaylova et al., "AMPK phosphorylates and inhibits SREBP activity to attenuate hepatic steatosis and atherosclerosis in diet-induced insulin-resistant mice," Cell Metabolism, vol. 13, no. 4, pp. 376-388, 2011.

[31] J. Zhou, M. X. Shi, T. D. Mitchell et al., "Changes in rat adipocyte and liver glucose metabolism following repeated restraint stress," Experimental Biology and Medicine, vol. 226, no. 4, pp. 312-319, 2001.

[32] M. Daval, F. Foufelle, and P. Ferré, "Functions of AMP-activated protein kinase in adipose tissue," The Journal of Physiology, vol. 574, no. 1, pp. 55-62, 2006. 
[33] J. Xiong, H. Yang, L. Wu et al., "Fluoxetine suppresses AMPactivated protein kinase signaling pathway to promote hepatic lipid accumulation in primary mouse hepatocytes," The International Journal of Biochemistry \& Cell Biology, vol. 54, pp. 236244, 2014.

[34] H. Liu, H. Zhong, Y. Yin, and Z. Jiang, "Genistein has beneficial effects on hepatic steatosis in high fat-high sucrose diet-treated rats," Biomedicine \& Pharmacotherapy, vol. 91, pp. 964-969, 2017.

[35] Y.-R. Yun, J.-H. Kim, J. H. Kim, and M. H. Jung, "Protective effects of gomisin $\mathrm{N}$ against hepatic steatosis through AMPK activation," Biochemical and Biophysical Research Communications, vol. 482, no. 4, pp. 1095-1101, 2017. 


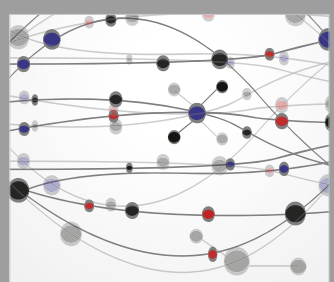

The Scientific World Journal
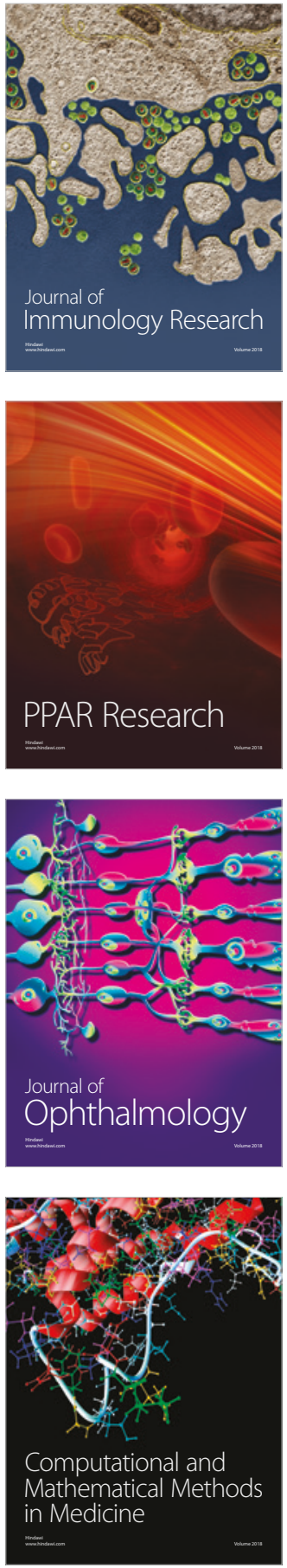

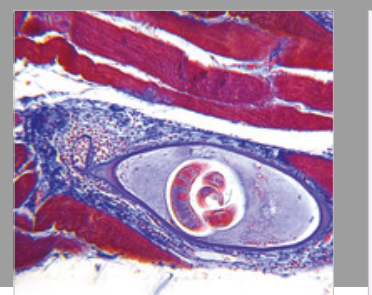

Gastroenterology Research and Practice

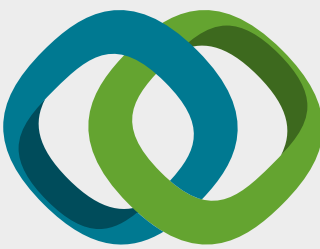

\section{Hindawi}

Submit your manuscripts at

www.hindawi.com
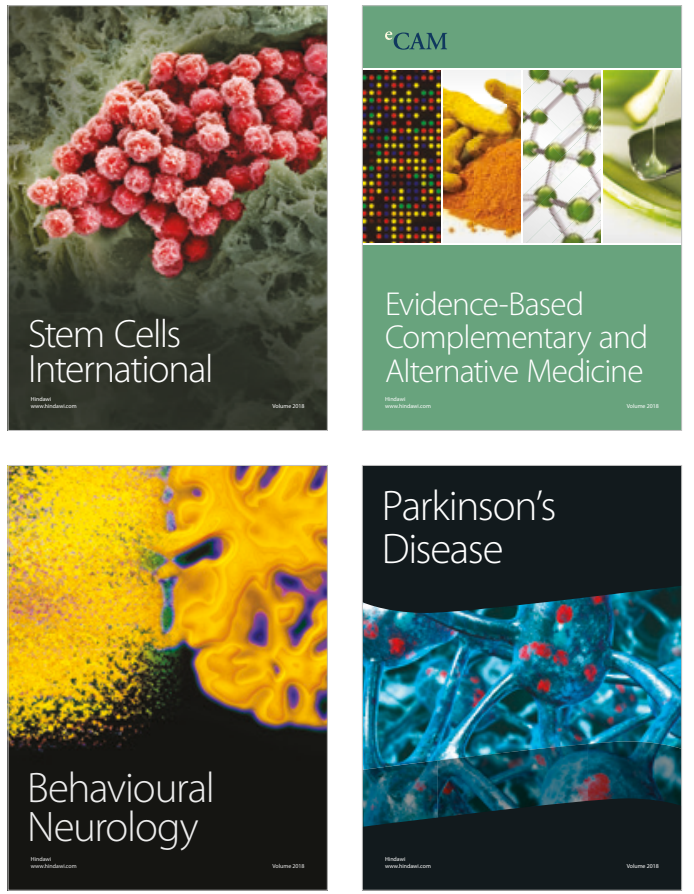

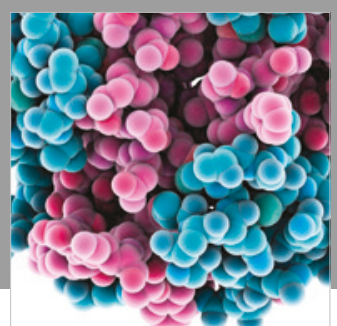

ournal of

Diabetes Research

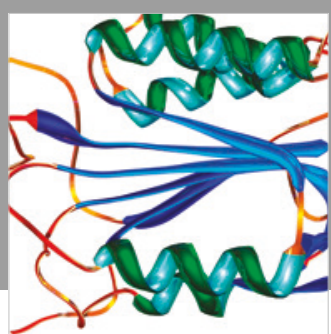

Disease Markers
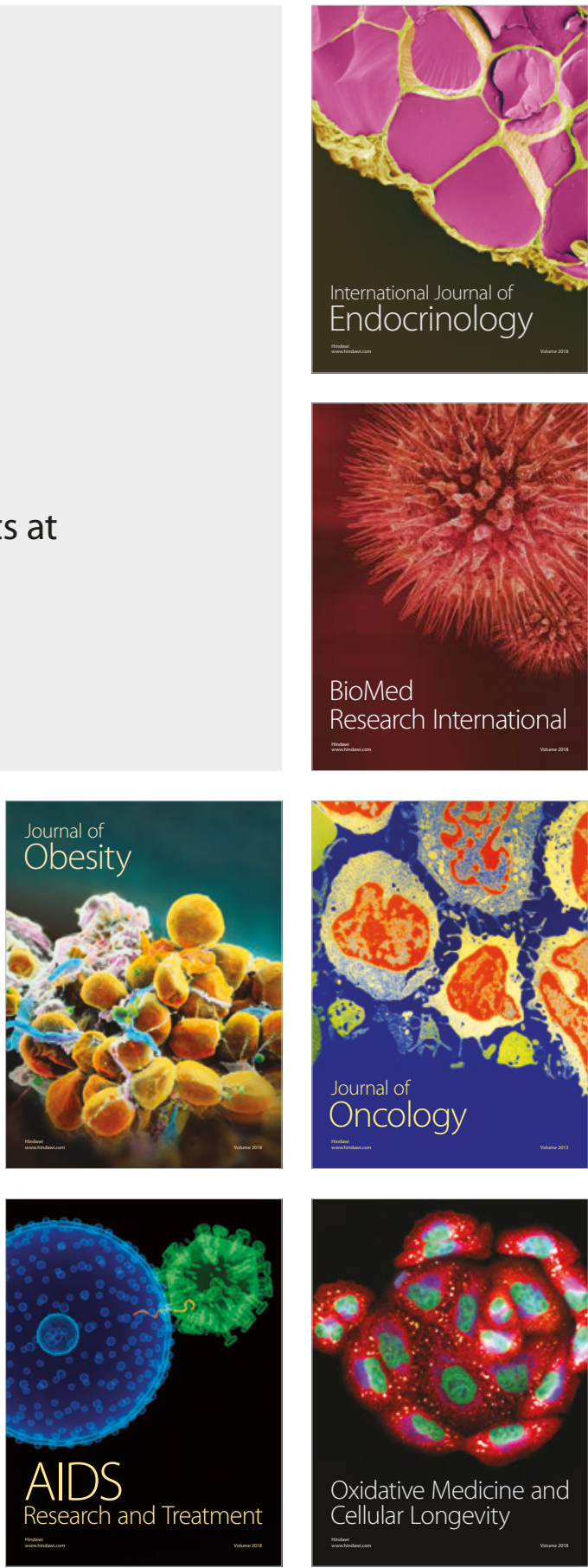